\title{
Information Sharing on Effects of Exposure to Building Materials in Adaptation Projects
}

\section{Sabarinah Sh Ahmad, Zarina Isnin, Zaharah Yahya, Mustapha Mohd Salleh}

Faculty of Architecture, Planning and Surveying

Universiti Teknologi MARA, Shah Alam 40450, Malaysia

sabar643@salam.uitm.edu.my

\begin{abstract}
Research findings confirm the existence of hazardous and toxic substances in most building materials. There is limited studies and information on building materials and the effects to health and safety. This study develops an evaluation method that is used to identify the presence of hazardous substances from building materials and assess their public health impact. The information allows stakeholders to identify potential risks and could improve knowledge sharing on building materials information on health and safety in the built environment industry.

Keywords: Building materials information; construction health and safety practices; hazardous substances; knowledge sharing

eISSN 2514-751X ๔ 2018. The Authors. Published for AMER ABRA cE-Bs by e-International Publishing House, Ltd., UK. This is an open-access article under the CC BY-NC-ND license (http://creativecommons.org/licenses/by$n c-n d / 4.0 /$ ). Peer-review under responsibility of AMER (Association of Malaysian Environment-Behaviour Researchers), ABRA (Association of Behavioural Researchers on Asians) and CE-Bs (Centre for EnvironmentBehaviour Studies), Faculty of Architecture, Planning \& Surveying, Universiti Teknologi MARA, Malaysia DOI: https://doi.org/10.21834/aje-bs.v3i10.313
\end{abstract}




\subsection{Introduction}

A large percentage of works undertaken nowadays were on improving the existing buildings such as refurbishment, renovation or building adaptation. The projects usually involve demolition or removal of existing building materials and installation of new finishes that may contain hazardous or toxic substance. The United Nations Conference on Environment and Development recognized that some building materials and construction activities can be harmful to human health (United Nations Environment Programme, 1992). Most innovative building materials involve the usage of chemicals. The use of chemicals has brought immense benefits to mankind, but some have also contributed to negative impacts on health and safety. Although there are increasing studies on building construction materials, information on health assessments of $95 \%$ of the chemicals used in building materials and construction environment is still insufficient (Pacheco-Torgal \& Labrincha, 2013). The construction sectors are also bogged with several other challenges on adequacy of information and knowledge on health risks posed by these man-made materials. Furthermore, the culture of knowledge sharing among construction workers especially in most developing countries is still restricted in traditional face-to-face interaction. Available information on the effects of building materials that could be understood by the stakeholders would assist them to share the knowledge and make better decisions (Isnin, Ahmad \& Yahya, 2012; Isnin, Ahmad \& Yahya, 2012b ).

This study aims to initiate the development of collecting information on effects of exposure to building materials as a knowledge base. The first objective examines through literature reviews on the needs of information on building materials that may risk human health and safety. The second objective develops an evaluation method that is used to identify the contents of building materials and assess their public health impact. Improving access to relevant information to the stakeholders involved would improve their knowledge and awareness on the effects of exposure to hazardous building materials.

\subsection{Information On Health And Safety Practices In Construction}

Information on hazards and risks is required before the construction work begins. The information can be disseminated through site briefings, meetings and distribution of paper medium. They should be made available to the workers or any stakeholders upon request. It may be in the form of hard copy or even digitised as long as the employees have access to the computer. According to the Uniited Nation, there is a need to provide information on the effects of building materials and the means available to mitigate their adverse health and safety impacts (Du Plessis, 2002). Many countries, such as the European Union (EU), the United States and most of the developed countries have already taken proactive actions to ensure the information on hazardous substances in building materials is accessible.

Building adaptation projects involve design, location, description, specification and quantity of different building materials from the existing space and for the new proposal. The work processes involve exposure to dust, fumes and liquids due to chipping, grinding, disassembling, attaching, sweeping and cleaning processes throughout the projects. None of the building materials specified in contemporary buildings is specifically designed to be 
healthful for people (McDonough \& Braungart, 2003). Few building materials used either for indoor or outdoor finishes have been tested for adverse health effects. Furthermore, studies have shown that some of the building materials used posed serious risks, such as asbestos and lead; exacerbated by the lack of adequate information on the existence, safe handling, and disposal of the debris. However, surveys on existing buildings seldom include information on hazardous materials (Isnin, Ahmad, \& Yahya, 2013). There were knowledge gaps on how changes in building design, construction, operation, occupancy and maintenance will influence the mix of pollutants from building materials and how the concentrations of indoor contaminants could be measured and affect human health (Isnin \& Ahmad, 2012; Isnin, Ahmad \& Yahya, 2012).

\subsection{Knowledge Gap on Building Materials Information}

Many chemicals previously thought to be safe are now found to have negative impacts on health and the environment. However, there are significant uncertainties in the reliability and validity of research findings on the effects of building materials on health. For example, data on the rate and frequency of emissions is scarce and some data is derived from inaccurate research methods (Benigeri, 2003). There is a lack of data on the identity of chemicals emitted, risks and on mitigation measures that could reduce exposures (Yashiro, 2011). Several studies reported inaccurate information on health and safety effects of exposure to building materials.

Building materials are often dismantled and removed without proper procedures. Construction wastes are seldom separated although they contain known hazardous substances such as asbestos. Occupational exposure to construction materials such as asbestos, lead, solvents and other hazardous materials is commonly unregulated with little monitoring of exposure. The working sites are often confined and congested. This has caused a great concern on the safety and health of workers. There was no chemical hazard specific to building materials identified, but the work site may still be exposed to certain risks derived from exposures to various chemical fumes, liquids, gases, vapours, mists and particulate matters.

\subsection{Knowledge Sharing Culture On Building Materials Information}

Information sharing among construction workers in developing countries is still restricted to traditional face-to-face interaction. The traditional knowledge sharing on building materials information is restricted to time, cost and quality of construction (Isnin et al., 2012c). Use of information technology such as computers, Internet or emails in construction companies is still piecemeal and limited in their daily work communication. Limited access to information resulted in lack of proper knowledge on how to deal with potential hazards.

Finding the appropriate information at the required time for an aggregated scale of building product information is a difficult task. Information may not be adequately managed due to lack of financial resources, technology and trained manpower. Information should be easy to understand for planning, decision making, construction processes, maintenance and as public information (Isnin et al., 2012b). There is a crucial need to ensure consultants, 
contractors and clients are provided with adequate information on safe work processes and mitigation strategies that enable them to avoid any potential hazards.

\subsection{Research Design}

The study explores some critical issues of building materials in building adaptation project through published secondary data. Samples for the study were building finishes of selected learning spaces in Klang Valley higher educational institutions in Malaysia. There were four groups of building materials involved in building adaptation work processes: demolish, dismantle, remains and new finishes. The first step of the study is to identify building materials for the projects. Specifications are defined according to the Economic Planning Unit guidelines. Standards are based on the Public Works Department, Malaysian Standards and other related standards and guidelines stipulated in the project documents. The data is grouped according to six building elements: (i) Wall and cladding, (ii) Floor, (iii) Ceiling, (iv) Door and accessories, (v) Window and accessories, and (vi) Built-in Furniture. Figure 1 shows the steps employed to identify potential risk and hazard of building materials in building adaptation projects.

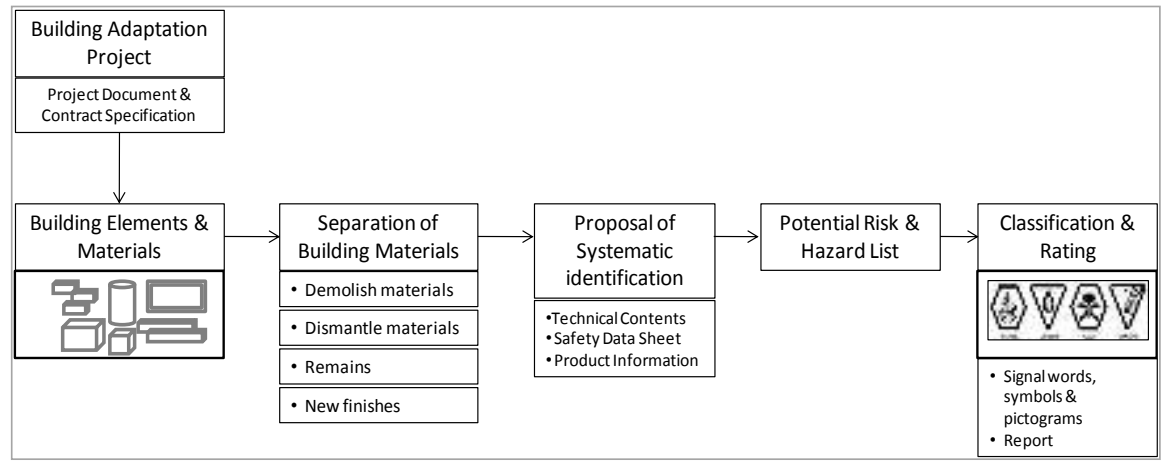

Fig. 1. Health and safety evaluation model for building materials in adaptation projects

\subsection{Proposal of systematic identification of building material substances}

The study recognised the need to identify ingredients used and the hazard identifications as specified by manufacturer or supplier. A reliable mechanism is needed to ensure the quality of information provided is accurate, reliable, valid and trustworthy. For this study, the information is based on Material Safety Data Sheets (MSDS) furnished by manufacturer or supplier, which is in accordance to Occupational Safety and Health (Classification, Packaging and Labelling of Hazardous Chemicals) Regulations 1997 under the Regulation 9. Normally each MSDS would have information on the product name and manufacturer's details, hazards identification, composition or information on ingredients, first aid measures, firefighting measures, accidental release measures, handling and storage, exposure controls, 
personal protection, physical and chemical properties, stability and reactivity, toxicological information, ecological information, disposal considerations, transport information, regulatory information and other information. Additional data, such as alternatives and cost data is also entered into the knowledge base.

It should be noted that accurate information is crucial for the study. MSDSs or technical specifications could be written by anyone and some may even be plagiarised (Supoh, 2005). An expert panel review conducted by OSHA in 1997 (Occupational Safety and Health Administration, n.d.), found that only $11 \%$ of the MSDSs were accurate in all of the following four areas: health effects, first aid, personal protective equipment, and exposure limits. The health effects data on the MSDSs are frequently incomplete and the chronic data are often incorrect or less complete than the acute data. Thus, any information is to be used with caution and should be obtained from trustworthy and reliable sources. The data and information, either from MSDS or other technical specifications, have to meet certain minimum standards of acceptability as outlined by the guidelines on preparation of MSDS and technical reports. The references should follow the required standards and formats. All information obtained is based on verifiable sources, for example, documented studies, reports, and reliable databases, as well as the currency of the report.

\subsection{List of potential risk and hazard}

Each building material examined is defined according to the "hazard identification" indicated on the MSDS. For example, one sample of wall paint is classified as "dangerous". It is further sub-classified according to the severity. Other building products could be classified as acute toxicity, flammability, etc. Other additional information also included the potential health effects and symptom through inhalation, ingestion, dermal contact, eye contact, chronic exposure and aggravation of pre-existing conditions. The hazard identification included physical, chemical and environmental hazards, if applicable.

\subsection{Indication of signal words and symbol/pictogram}

The model uses symbol or pictogram to enable the users to recognize and understand the hazard and risk. These symbols/pictograms consist of a black hazard symbol surrounded by a red frame as per standards. However, the requirements for the use of pictograms depend on hazard class as well as hazard category. The style of language and presentation is presented using less technical terms, as many of the users may not be familiar with the technical jargons.

\subsection{Limitations}

Some of the information on the existing building materials was not available or incomplete. There were also unrecorded changes for the finishes due to repair works or maintenance. Some information on details of secondary materials or building products such as cornices or the adhesives and glues used was not available as they are often unrecorded. Thus, references are made based on the detail drawings or installation guidance. The study also excludes loose furniture such as chairs and tables at the research site. 


\subsection{Findings and Discussion}

Twenty nine types of generic building finishes and related components were examined based on the six building elements of a typical classroom in public higher educational institutions. There were many manufacturers of these finishes and components. A search of the MSDS database for each finishes and components resulted in 358 MSDSs that are coded for easier referencing and retrieval. There were several similar specifications for similar products but from different manufacturers. Sometimes they were of different formulation and came with different safety recommendations. The information is further categorised and entered into a knowledge base. Additional data such as alternative materials is obtained from alternative reliable sources to demonstrate the availability of choice. Acts, guidelines and specifications related to the building products are included for references.

\subsection{What Information Can Be Shared?}

From the proposed model, knowledge could be obtained from the collected data and information on the MSDSs and other technical information. This knowledge repository would assist the stakeholders to make decisions or show the right way of doing a task or preventive methods. The knowledge could also be shared amongst the different users. Based from Fig. 2 , specific information on potential risk and hazard from building materials used in projects could be identified.

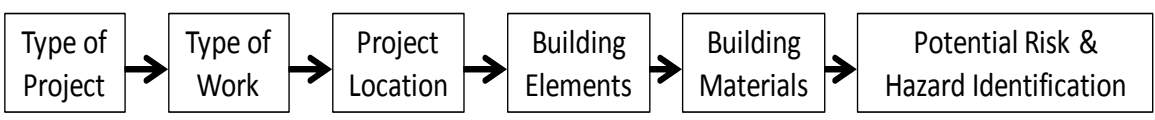

Fig. 2. Information on potential risk and hazard identification form building materials used in a specified project

The knowledge repository on the existing building materials would inform and alert the stakeholders on the presence of a variety of potential sources such as:

- Existing materials on site based from previous design specifications such as asbestos ceiling sheets, lead paints, polyvinyl chloride (PVC) etc.,

- Materials that are incorporated in existing building components, such as asbestos in insulation etc.,

- Work processes, which could change stable or inert materials into hazardous form, such as cutting hard concrete, gluing adhesives in confined space etc.,

- New materials specified by the designer for the project that could contain hazardous substances, and

- Contaminations due to dampness, molds, lack of maintenance that could change the chemical form of materials.

Models and simulations of the proposed system could provide crucial information on the potential risk and hazards of building materials in construction projects. The model generates 
a preliminary hazard list for building substances that could assist design, construction, maintenance and occupancy throughout the building life-cycle development.

\subsection{Information On Potential Health Effects And Preventive Measures}

The specific data collection and retrieval efforts from the model are defined under the health and safety data and monitoring requirements (Figure 3). Other related activities and additional information on building materials may assist in eliminating or reducing the potential risks and hazards on site (Fig. 4). These information need to be accurate and valid. The information is based on sound technical, scientific or engineering judgement provided by reliable sources.

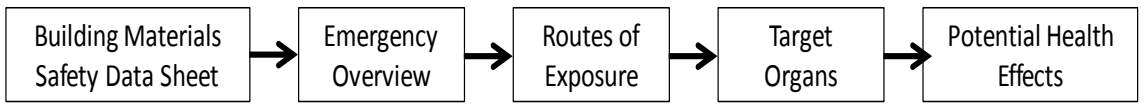

Fig. 3. Information on potential health effects from exposure to building materials

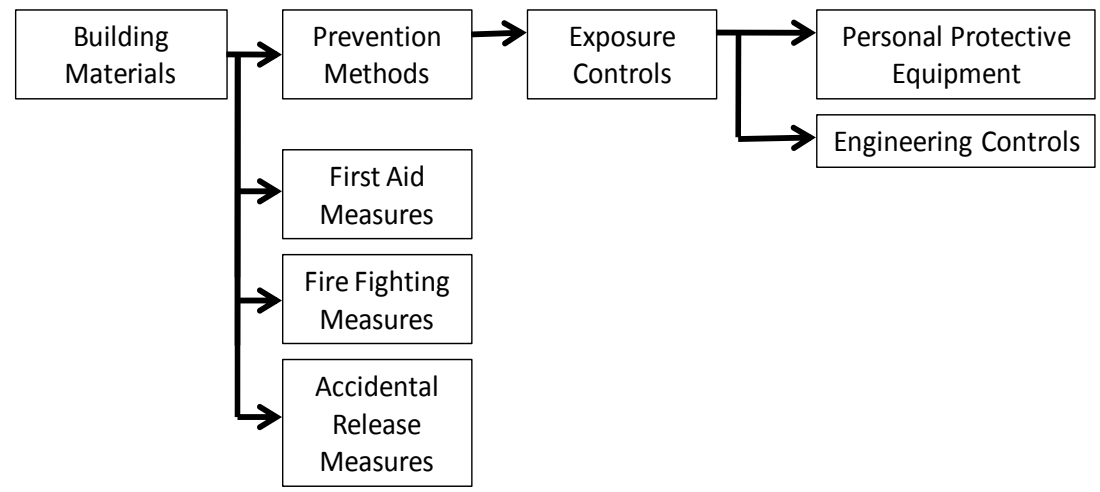

Fig. 4. Information on preventive measures

\subsection{Information On Alternative Building Materials}

For the proposed model, it proposes information on suitable alternatives such as "Green" certified materials (Fig. 5). The proposed model attempts to help assessment and comparison of available building product relative to another.

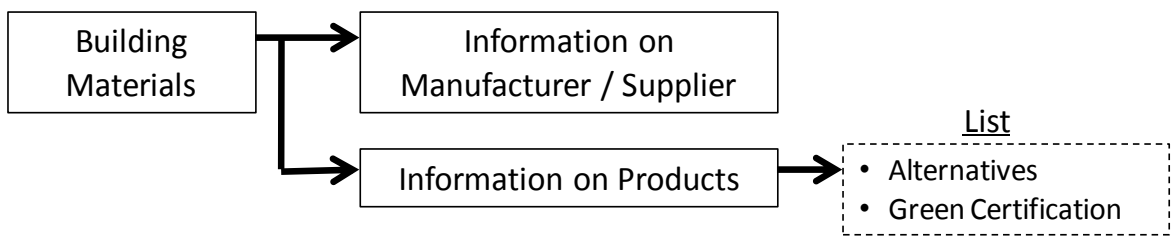

Fig. 5. Information on alternative buidling materials 


\subsection{Knowledge Repository On Building Materials}

The proposed model defines the tasks and implications of exposure to building materials throughout the lifecycle of the building (Fig. 6). The information can be applied to different phases of a project. The model may assist planning, organising, controlling and managing the exposure to any potential hazardous building substances. Usage of appropriate information system would assist rapid search and retrieval of information. It is also more efficient compared with a search in unstructured database system.

This database inventory may allow stakeholders to identify potential hazardous building materials that may damage health and the environment. The knowledge is then shared amongst stakeholders to properly manage and monitor the safe use in construction processes.

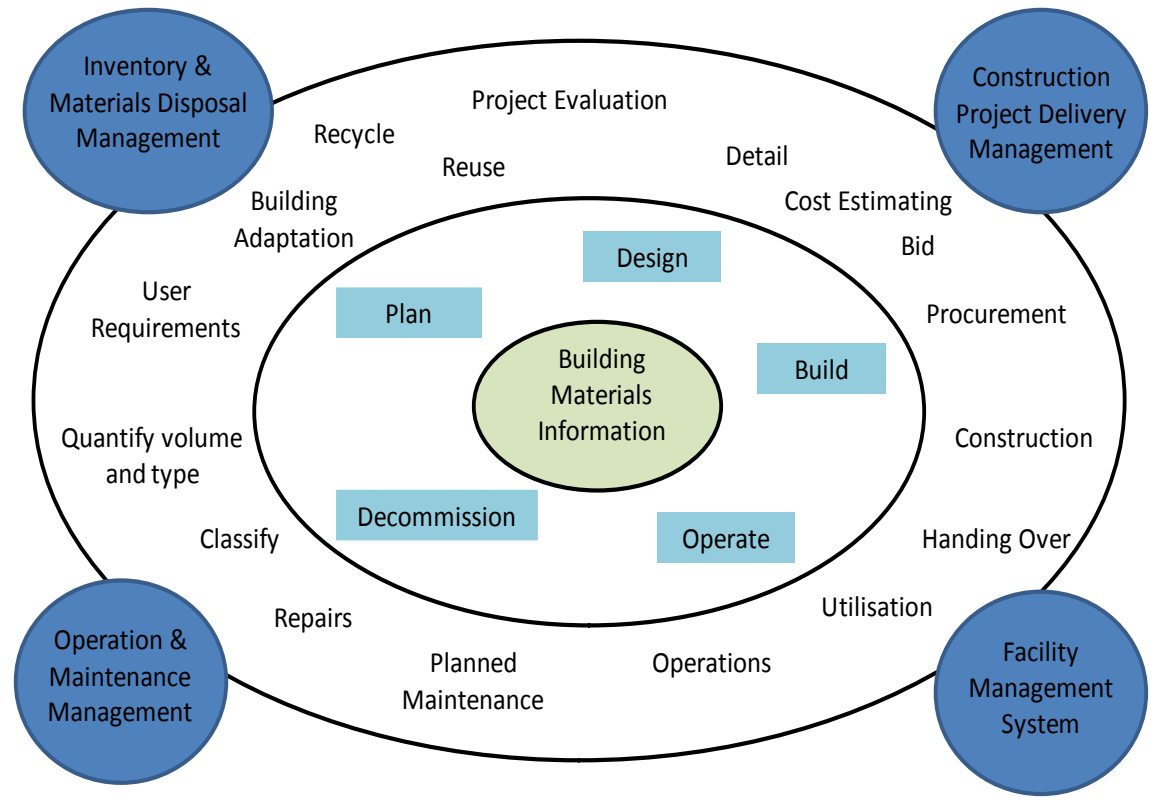

Fig. 6. Collection of database on building materials throughout the building lifecycle suitable for a knowledge repository.

(Adapted from Building Information Framework building informationmanagement.wordpress.com)

\subsection{Conclusion and Recommendation}

Chemicals incorporated in building materials have caused adverse effects to human health and the environmental sustainability. There is a need for improved availability of research findings that could be shared amongst the stakeholders. Information on building materials should be collected and kept in a knowledge repository. The knowledge could be shared and transmitted to the stakeholders for better decision makings. They would be able to identify 
potential harmful materials and reduce any adverse effects. There are choices of less harmful building material alternatives to suit the project. The shared knowledge on building materials information may be the greatest investments to health and safety in the built environment industry.

\section{Acknowledgement}

The authors would like to thank Research Management Institute of Universiti Teknologi MARA for supporting this research under the Excellence Research and the Research Intensive Faculty grant schemes.

\section{References}

Benigeri, M. (2003). Shortcomings of health information on the Internet. Health Promotion International, 18(4), $381-$ 386. doi:10.1093/heapro/dag409

Du Plessis, C. (2002). Agenda 21 for sustainable construction in developing countries - A discussion document (No. Boutek Report No Bou/E0204; ISBN 0-7988-5540-1). Construction. South Africa.

Isnin, Z., Ahmad, S. S., Yahya, Z. (2013). Lessons Learned from Exposure to Building Materials. Procedia-Social and Behavioral Sciences, 85(2013), 128-138

Isnin, Z., \& Ahmad, S. S. (2012). Challenges and the Way Forward for Building Materials Management in Building Adaptation Projects. Advanced Materials Research, 488-489, 274-278. doi:10.4028/www.scientific.net/AMR.488489.274

Isnin, Z., Ahmad, S. S., Yahya, Z. (2012). Awareness and Knowledge of the Hidden Killers in Building Adaptation Projects. Procedia-Social and Behavioral Sciences, 68(2012), 43-52

Isnin, Z., Ahmad, S. S., Yahya, Z. (2012b). Challenges of the Unknown Building Material Substances for Greener Adaptation Projects. Procedia-Social and Behavioral Sciences, 68(2012), 53-62

McDonough, W., \& Braungart, M. (2003). Toward a Sustaining Architecture for the 21st Century:The Promise of Cradle-to-Cradle Design. Industry \& Environment, 26(2-3). Retrieved from http://www.mcdonough.com/speakingwriting/toward-a-sustaining-architecture-for-the-21st-centurythe-promise-of-cradle-to-cradle-design/

Occupational Safety and Health Administration. (n.d.). Hazard Communication in the 21st Century Workplace. Retrieved January 11, 2012, from http://www.osha.gov/dsg/hazcom/finalmsdsreport.html

Pacheco-torgal, F., \& Labrincha, J. A. (2013). The future of construction materials research and the seventh UN Millennium Development Goal: A few insights. Construction and Building Materials, 40, 729-737. doi:10.1016/j.conbuildmat.2012.11.007

Supoh, H. (2005). A Current Status of GHS Implementation in Malaysia in Regional Workshop on Chemical Hazard Communication and GHS Implementation for Countries of the Association of Southeast Asian Nations (ASEAN). , GHS ASEAN Workshop, Regional workshop on chemical hazard communication and GHS implementation for countries of the Association of Southeast Asian Nations (ASEAN), Manila, Philippines. Manila, Philippines. 
Sh Ahmad, S., et.al. / Asian Journal of Environment-Behaviour Studies (ajE-Bs), 3(10) Sep / Oct 2018 (p58-67)

United Nations Environment Programme. (1992). Rio Declaration on Environment and Development The United Nations Conference on Environment and Development ,. Environment (pp. 14-16). Retrieved from www.cfam.org/.../20080625_Rio_Declaration_on_Environment.pdf

Yashiro, T. (2011). The need for effective reporting on sustainable buildings: translating to policy. In UNEP-SCBI Symposium on Sustainable Buildings. Leverkusen, Germany. 